\title{
Right-sided endocarditis in an adult patient with hyperimmunoglobulin-E syndrome successfully treated with high-dose daptomycin
}

\author{
Natalia G. Vallianou, Penny Gounari, Alex Skourtis, J ohn Panagos, E. Sioula \\ Department of Internal Medicine, Evangelismos General Hospital, Athens, Greece \\ Correspondence: Natalia Vallianou. Address: 5 Pyramidon str, 190 05, Municipality of Marathon, Athens, Greece. Email: \\ natalia.vallianou@hotmail.com
}

Received: February 13, 2014

Accepted: March 12, 2014

Online Published: March 24, 2014

DOI : $10.5430 /$ crim.v1n2p51

URL: http://dx.doi.org/10.5430/crim.v1n2p51

\begin{abstract}
Hyperimmunoglobulin-E syndrome is a syndrome of recurrent staphylococcal abscesses, sinopulmonary infections, and severe eczema, which is characterized by increased IgE and specific facial features. Herein, we describe a patient with hyperimmunoglobulin-E syndrome and right-sided endocarditis due to Staphylococcus aureus, which was successfully treated with high-dose daptomycin.
\end{abstract}

\section{Keywords}

Staphylococcus, Tricuspid valve endocarditis, Daptomycin, Hyperimmunoglobulin-E syndrome

\section{I ntroduction}

Hyperimmunoglobulin-E syndrome (HIES) is a syndrome of recurrent staphylococcal abscesses, sinopulmonary infections, severe eczema and elevated levels of serum $\operatorname{IgE}^{[1,2]}$. Herein, we describe a patient with HIES and right-sided endocarditis due to Staphylococcus aureus.

\section{Case presentation}

A forty-two years old male patient presented to the hospital with fever and rigors of one week's duration. On examination, the patient appeared acutely ill, with dyspnea, edema of both legs, crackles on lung auscultation, a temperature of $40^{\circ} \mathrm{C}$ and tachycardia. A transthoracic cardiac triplex, which was performed due to signs of right-heart failure, revealed the presence of a vegetation in the tricuspid valve, with concomitant major tricuspid valve regurgitation (see Figure 1). The patient was admitted to the hospital.

From his past medical history, he was suffering from eczema and had many respiratory tract infections in the past, some of which required admission to the hospital. He denied being an intravenous drug user and he had been working as a manager in a big company. 
Five out of five blood cultures yielded Staphylococcus aureus, sensitive to methicillin and the presence of right-heart endocarditis was documented. Dicloxacillin $2 \mathrm{~g}$ every six hours was administered. Due to the presence of crackles on both lungs, a spiral CT was performed, which revealed the presence of multiple septic emboli as well as pleuritic fluid, that was compatible with an exudate (see Figure 2). As the patient remained febrile while receiving oxacillin, the regimen was changed to high-dose daptomycin and rifampin. Vancomycin was not administered to the patient due to impaired renal function, which was attributed to glomerulonephritis. As daptomycin has been documented to be successful in the treatment of right-sided endocarditis, it was chosen instead of vancomycin because it seems not to worsen renal function. With the administration of daptomycin and rifampin for endocarditis, the patient gradually became afebrile and he was discharged from hospital having only mild edema in the legs and no crackles in the lungs.

Due to the presence of right-sided endocarditis, which is much more common in intravenous drug users than in the general population, we began to explore what could have been responsible for the right-sided endocarditis in this patient. Due to its medical history of many respiratory tract infections and eczema, the presence of HIES was suspected, which was confirmed with the finding of serum IgE levels of $1776 \mathrm{UI} / \mathrm{L}$. Thus, the presence of HIES could account for the right-sided endocarditis due to Staphylococcus aureus in this patient.

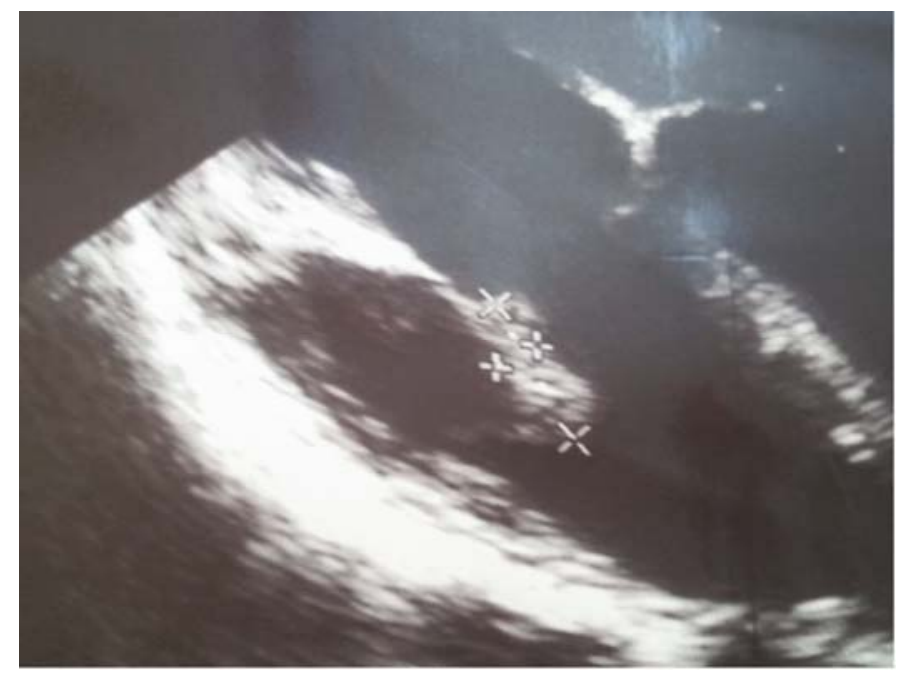

Figure 1. The tricuspid's valve vegetation sizing $18.8 \mathrm{~mm} \times 10.2 \mathrm{~mm}$

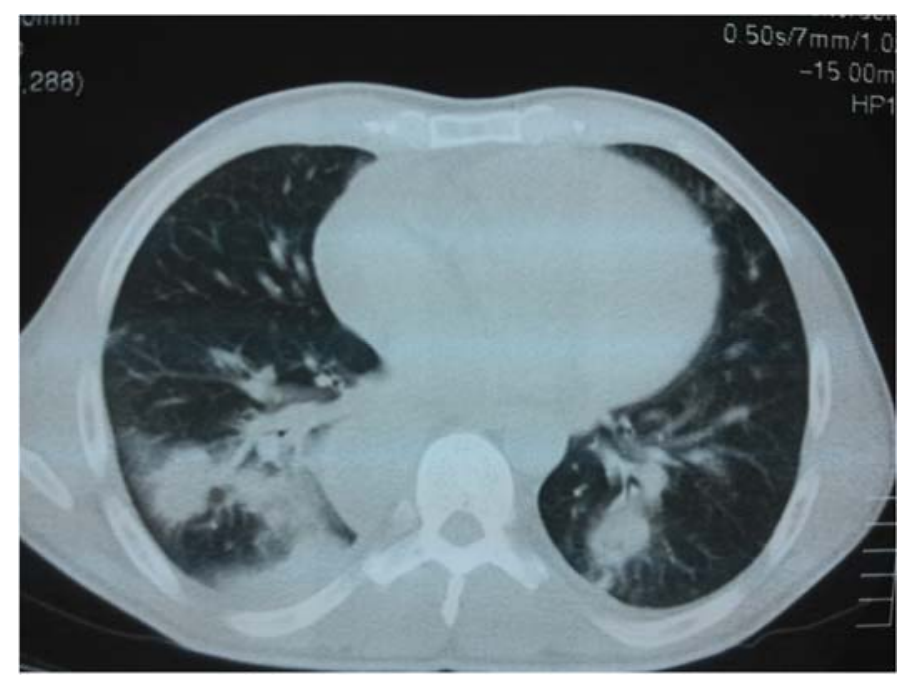

Figure 2. Spiral CT scan depicting multiple septic emboli presenting as lung abscesses. 


\section{Discussion}

HIES, which was first described in 1966 and was called Job's syndrome, is characterized by specific facial characteristics, together with the presence of increased IgE levels, eczema and recurrent infections, which are usually caused by Staphylococcus aureus ${ }^{[2-5]}$. The most characteristic facial feature is a broad nasal base and broad nasal bridge, protrusion of the forehead, wide outer canthal distances, and deep set eyes ${ }^{[6,7]}$.

HIES is due to defects in Janus activated kinase/signal transducer and activator of transcription (JAK-STAT)-mediated cytokine signals, including interleukin (IL)-6 and IL-23 ${ }^{[8-13]}$.

To our knowledge, right-sided endocarditis has only once been reported before in a patient with HIES ${ }^{[14]}$. It seems likely that Staphylococcus aureus found its way to cause bacteremia and tricuspid valve endocarditis through the eczematous skin of the patient. The defective immune response due to the presence of the HIES could account for the presentation with right-sided endocarditis and multiple pulmonary emboli. It is also noteworthy that the patient responded well to high-dose daptomycin as defervescence occurred, only after having switched the antimicrobial chemotherapy from oxacillin to daptomycin.

\section{References}

[1] Davis SD, et al. Syndrome. Recurrent, “cold”, staphylococcal abscesses. Lancet. 1966; 1: 1013. http://dx.doi.org/10.1016/S0140-6736(66)90119-X

[2] Hill HR, Quie PG. Raised serum-IgE levels and defective neutrophil chemotaxis in three children with eczema and recurrent bacterial infections. Lancet. 1974; 1: 183. http://dx.doi.org/10.1016/S0140-6736(74)92493-3

[3] Hill HR, et al. Defect in neutrophil granulocyte chemotaxis in Job's syndrome of recurrent "cold" staphylococcal abscesses. Lancet. 1974; 2: 617. http://dx.doi.org/10.1016/S0140-6736(74)91942-4

[4] Grimbacher B, et al. Hyper-IgE syndrome with recurrent infections--an autosomal dominant multisystem disorder. N Engl J Med. 1999; 340: 692. http://dx.doi.org/10.1056/NEJM199903043400904

[5] Grimbacher B, Holland SM, Puck JM. Hyper-IgE syndromes. Immunol Rev. 2005; 203: 244. http://dx.doi.org/10.1111/j.0105-2896.2005.00228.x

[6] Minegishi Y, Karasuyama H. Defects in Jak-STAT-mediated cytokine signals cause hyper-IgE syndrome: lessons from a primary immunodeficiency. Int Immunol. 2009; 21: 105. http://dx.doi.org/10.1093/intimm/dxn134

[7] Heimall J, Freeman A, Holland SM. Pathogenesis of hyper IgE syndrome. Clin Rev Allergy Immunol. 2010; $38: 32$. http://dx.doi.org/10.1007/s12016-009-8134-1

[8] Minegishi Y, et al. Dominant-negative mutations in the DNA-binding domain of STAT3 cause hyper-IgE syndrome. Nature. 2007; 448: 1058. http://dx.doi.org/10.1038/nature06096

[9] Holland SM, et al. STAT3 mutations in the hyper-IgE syndrome. N Engl J Med. 2007; 357: 1608. http://dx.doi.org/10.1056/NEJMoa073687

[10] Minegishi Y, Karasuyama H. Defects in Jak-STAT-mediated cytokine signals cause hyper-IgE syndrome: lessons from a primary immunodeficiency. Int Immunol. 2009; 21: 105. http://dx.doi.org/10.1093/intimm/dxn134

[11] Al Khatib S, et al. Defects along the $\mathrm{T}(\mathrm{H}) 17$ differentiation pathway underlie genetically distinct forms of the hyper IgE syndrome. J Allergy Clin Immuno. 2009; 124: 342. http://dx.doi.org/10.1016/j.jaci.2009.05.004

[12] Van de Veerdonk FL, et al. Milder clinical hyperimmunoglobulin E syndrome phenotype is associated with partial interleukin-17 deficiency. Clin Exp Immunol. 2010; 159: 57. http://dx.doi.org/10.1111/j.1365-2249.2009.04043.x

[13] Chandesris MO, et al. Autosomal dominant STAT3 deficiency and hyper-IgE syndrome: molecular, cellular, and clinical features from a French national survey. Medicine (Baltimore). 2012 Jul; 91(4): e1-19. http://dx.doi.org/10.1097/MD.0b013e31825f95b9

[14] Gupta S, Mittal A, Gupta Jagdish. Tricuspid endocarditis in hyperIgE syndrome. J Postgrad Med. 2010; 56(2): $143-145$. http://dx.doi.org/10.4103/0022-3859.65293 